\title{
Presence of Postsystolic Shortening Increases the Likelihood of Coronary Artery Disease: A Rest Electrocardiography-Gated Myocardial Perfusion SPECT Study
}

\author{
Yumiko Kanzaki ${ }^{1}$, Yohei Yamauchi ${ }^{1}$, Hideaki Morita ${ }^{1}$, Masuo Hayashi $^{2}$, Tsuyoshi Komori ${ }^{2}$, Akira Ukimura ${ }^{1}$, \\ and Nobukazu Ishizaka ${ }^{1}$ \\ ${ }^{I}$ Department of Cardiology, Osaka Medical College, Takatsuki, Japan; and ${ }^{2}$ Department of Radiology, Osaka Medical College, \\ Takatsuki, Japan
}

\begin{abstract}
Postsystolic shortening (PSS), which is a delayed myocardial contraction that occurs after end-systole, has been considered an important diagnostic index of myocardial ischemia. Recent technological advancements in quantitative gated SPECT (QGS) software enables the left ventricular (LV) regional analysis and may be useful for PSS measurement. The purpose of this study was to evaluate whether PSS at the resting condition determined by QGS is useful to identify patients with coronary artery disease. Methods: The study comprised 146 patients (mean age $\pm \mathrm{SD}, 71 \pm 8 \mathrm{y}$; 98 men) with normal LV wall motion (mean LV ejection fraction \pm SD, $72 \% \pm 9 \%$ ) who underwent both coronary angiography and resting $99 \mathrm{mTC}-$ tetrofosmin myocardial perfusion SPECT. The sum of the difference between post-end-systolic maximal LV thickening and end-systolic LV thickening, designated PSS index, was calculated from 17 LV myocardial segments using QGS. Results: The PSS index was significantly higher in patients with significant stenosis of the coronary artery than in the other patients $(9.8 \pm 10.2$ vs. $5.6 \pm 5.1 ; P<0.01)$. A cutoff point of 6.0 of the PSS index had sensitivity, specificity, positive predictive value, and negative predictive values of $55 \%$, $70 \%, 76 \%$, and $47 \%$, respectively, for the diagnosis of coronary artery disease. Multivariate logistic regression analysis demonstrated that a PSS index greater than 6.0 was an independent predictor for the presence of coronary artery disease (odds ratio, 2.46; 95\% confidence interval, 1.1-5.4; $P<0.05$ ). Conclusion: Among subjects with normal LV function, PSS index even in the resting condition determined using QGS may help to identify patients with coronary artery disease.
\end{abstract}

Key Words: coronary artery disease; scintigraphy; postsystolic shortening

J Nucl Med 2015; 56:1889-1894

DOI: 10.2967/jnumed.115.153791

Q uantitative electrocardiography-gated SPECT myocardial perfusion imaging (quantitative gated SPECT [QGS]) can be used to acquire the left ventricular (LV) time volume curve at several

Received May 26, 2015; revision accepted Aug. 31, 2015.

For correspondence or reprints contact: Yumiko Kanzaki, Department of Cardiology, Osaka Medical College, 2-7, Daigaku-machi, Takatsuki 5698686, Japan.

E-mail: in3089@poh.osaka-med.ac.jp

Published online Sep. 10, 2015.

COPYRIGHT (c) 2015 by the Society of Nuclear Medicine and Molecular Imaging, Inc. phases of the cardiac cycle and, therefore, to assess LV wall motion $(1-5)$. Advances in QGS software have enabled the analysis of the LV regional wall motion (6-8). Several previous studies have used this method to investigate the diagnostic and prognostic values of LV dyssynchrony in patients with heart failure (9-13) and ischemic heart disease (14-17). Postsystolic shortening (PSS), defined as delayed myocardial contraction occurring after end-systole, is observed in patients with flow-limiting coronary stenosis and has also been proposed as a marker of myocardial ischemic memory (18-26).

The LV wall motion can be analyzed by QGS using an automated procedure with high reproducibility. In the present study, we used QGS to investigate whether the presence of PSS at rest is associated with an increased likelihood of coronary artery stenosis in subjects with normal global LV systolic function.

\section{MATERIALS AND METHODS}

\section{Study Patients}

We retrospectively analyzed data from 146 consecutive patients (98 men, 48 women; mean age $\pm \mathrm{SD}, 71 \pm 8$ y) who underwent myocardial perfusion SPECT for the diagnosis of active myocardial ischemia because of the presence of signs or symptoms suggestive of myocardial ischemia or to the presence of proven coronary artery lesions, who also underwent coronary artery angiography within 3 mo before and after the myocardial perfusion SPECT study. Exclusion criteria included decompensated heart failure, atrial fibrillation, history of heart surgery, previous myocardial infarction, cardiomyopathy, advanced arterial ventricular block, severe chronic obstructive pulmonary disease, bronchospasm, ejection fraction less than 55\%, left bundle branch block, and postpacemaker implantation. The institutional review board (the ethical committee of the Osaka Medical College) approved this retrospective study, and the requirement to obtain informed consent was waived.

\section{SPECT Imaging}

Gated SPECT myocardial perfusion imaging was routinely performed using a 1-d stress-rest protocol that included physical exercise with a bicycle ergometer (RH-32; Lode Instrumenten) or administration of adenosine (Daiichi Sankyo Pure Chemicals). ${ }^{99 \mathrm{~m} T c-t e t r o f o s m i n}$ (296 MBq) was intravenously injected $1 \mathrm{~min}$ before near-maximum stress or $3 \mathrm{~min}$ after the start of continuous adenosine infusion. Twelve-lead electrocardiograms and blood pressure measurements were obtained at baseline and every minute during the stress test. Myocardial SPECT acquisition was performed 30-45 min later after injection. Four hours later, resting SPECT was performed approximately $1 \mathrm{~h}$ after an injection of $600 \mathrm{MBq}$ of ${ }^{99 \mathrm{~m}} \mathrm{Tc}$-tetrofosmin.

A triple-detector SPECT system (GCA-9300A/PI; Toshiba Medical Systems) equipped with a high-resolution parallel-hole collimator was 
used for the imaging studies. The gated SPECT data were acquired using the following parameters: $360^{\circ}$ step-and-shoot rotation, 60 steps per head, $60 \mathrm{~s}$ per step, $128 \times 128$ matrix $(1$ pixel $=3.2 \mathrm{~mm}), 16$ frames per cardiac cycle, and a beat acceptance window at $20 \%$ of the average R-R interval calculated just before the data collection began. The ungated projection data were obtained by summing the gated projection data. The $360^{\circ}$ projection data were reconstructed by performing filtered backprojection with a Butterworth filter (cutoff frequency, 0.47 cycles/cm; order, 8) using a GMS-7700B workstation (Toshiba Medical Systems). The reconstructed short-axis images were analyzed using quantitative perfusion SPECT with QGS software (Cedars-Sinai Medical Center). The ejection fraction, end-diastolic volume, and end-systolic volume were automatically obtained from the QGS analysis.

\section{Phase Analysis and PSS Index Measurements}

Phase analysis of the left ventricle was performed on the resting electrocardiography-gated SPECT dataset using QGS 2007 software (6) on a GMS-7700B workstation. The global and regional dyssynchrony measures were automatically computed. For this study, a histogram was constructed from the phase values for the entire left ventricle, and phase $\mathrm{SD}$ and histogram bandwidth were calculated and examined as the global dyssynchrony measures (6-16). Timing values were expressed in degrees $\left(0^{\circ}-360^{\circ}\right)$. End-systole was defined using the total average thickening curve, and 17-segment thickening curves were drawn. The time to maximal (max) LV thickening (msec), max LV thickening $(\mathrm{mm})$, and end-systolic LV thickening $(\mathrm{mm})$ were measured for each of the 17 segments. The PSS index was derived from the sum of the difference between post-end-systolic max LV thickening and endsystolic LV thickening divided by post-end-systolic max LV thickening from the 17 segments (21). If a segment curve showed that the time to max LV thickening occurred before end-systole, the difference between max LV thickening and end-systolic LV thickening was assumed to be 0 (Fig. 1).

\section{Evaluation of Perfusion}

The SPECT images from the poststress and resting studies were displayed on a personal computer monitor. The images were generated both in gray scale and in color scale from the raw transaxial tomograms. Orthogonal images were generated using oblique angle reconstruction, producing short-axis, horizontal long-axis, and vertical long-axis views. In addition, polar maps were generated to enable comparison of global perfusion. Two experienced observers with no knowledge of the clinical data examined the images and estimated the degree of myocardial ischemia. The diagnostic accuracy was evaluated using patient-based data. A 17-segment model was used to categorize perfusion defects. Individual segments were scored as follows: 0 , normal; 1 , mildly reduced; 2 , moderately reduced; 3 , severely reduced; and 4 , absent. The severity of reversible defects in myocardial perfusion was defined on the basis of the summed difference scores (SDSs) derived from summed stress scores (SSSs) and summed rest scores (SRSs) for all the segments. For the calculation of sensitivity, specificity, and predictive values for angiographic coronary artery disease, an interpretive score or greater was defined as abnormal in the overall territory. Differences between the interpreters were resolved by consensus.

\section{Coronary Angiography}

The interval between coronary angiography and myocardial perfusion SPECT was less than 3 mo. The degree of coronary artery stenosis was visually rated according to the criteria of the American Heart Association by an experienced investigator unaware of the SPECT data (27). Significant stenosis was considered to be present when $75 \%$ or more narrowing of the diameter was observed within any of 13 segments $(1-4,5-9$, and 11-14).

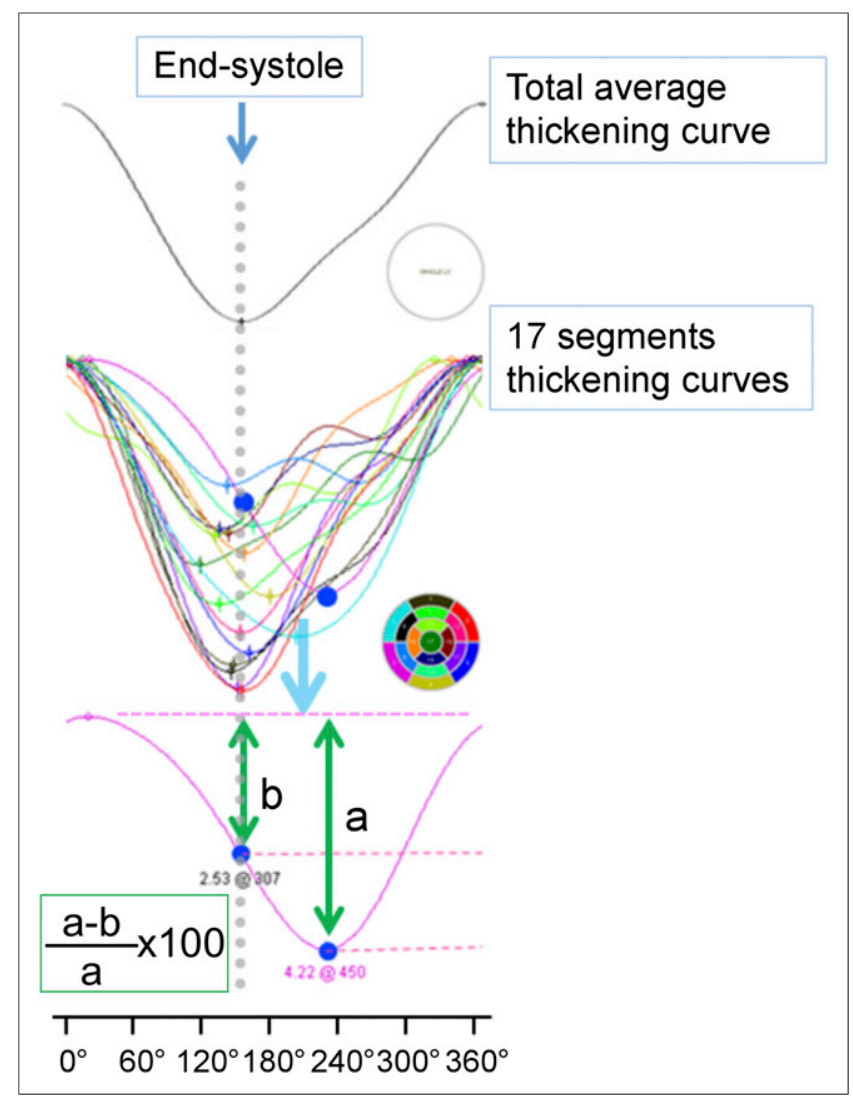

FIGURE 1. Measurements of PSS index. (Upper) End-systole was defined on the basis of total average thickening curve. (Lower) PSS was determined from 17 segments using sum of difference between post-endsystolic max LV thickening (a) and end-systolic LV thickening (b) divided by post-end-systolic max LV thickening (a). If segment curve showed time to max LV thickening before end-systole, difference between max LV thickening and end-systolic LV thickening was assumed to be 0 .

\section{Data Analysis}

The differences in parametric data between the 2 groups were tested using the Student $t$ test for normally distributed data (i.e., age, body mass index, end-diastolic volume, ejection fraction, and transient ischemic dilatation) or the Kruskal-Wallis test for data not normally distributed data (i.e., end-systolic volume, phase SD, histogram bandwidth, PSS index, SSS, SRS, and SDS). The findings were expressed as mean \pm SD. Sensitivity, specificity, positive predictive value (PPV), and negative predictive value (NPV) in indices as predictors of significant coronary artery disease were calculated in the traditional manner. Receiveroperating-characteristic (ROC) curves were analyzed to determine the optimal cutoffs for the SSS, SRS, SDS, phase SD, histogram bandwidth, and PSS index. Univariate or stepwise multivariate logistic regression analysis was used to identify coronary artery disease. Proportions of diagnostic accuracy were compared using the $\chi^{2}$ test when appropriate. A $P$ value of less than 0.05 was considered statistically significant. Data analysis was performed using SPSS (version 22.0; IBM/SPSS).

\section{RESULTS}

The characteristics of the study patients are shown in Table 1. Coronary angiographic examination revealed that 53 of the 146 patients did not have significant coronary artery stenosis, 57 had single-vessel disease, and 36 had multivessel disease. There was no significant difference in either of the 2 conventional 
TABLE 1

Patient Characteristics

\begin{tabular}{|c|c|c|c|}
\hline Characteristic & $\begin{array}{l}\text { No coronary artery } \\
\text { disease }(n=53)\end{array}$ & $\begin{array}{l}\text { Coronary artery } \\
\text { disease }(n=93)\end{array}$ & $P$ \\
\hline Mean age $\pm S D(y)$ & $70.5 \pm 8.3$ & $70.6 \pm 8.1$ & NS \\
\hline Male sex $(n)$ & $29(55)$ & $69(74)$ & 0.02 \\
\hline Exercise & $21(40)$ & $47(51)$ & NS \\
\hline Adenosine & $32(60)$ & $46(49)$ & NS \\
\hline Body mass index & $23.7 \pm 3.1$ & $24.1 \pm 3.0$ & NS \\
\hline Hypertension (n) & $47(89)$ & $83(89)$ & NS \\
\hline Dyslipidemia (n) & $33(62)$ & $67(72)$ & NS \\
\hline Diabetes mellitus $(n)$ & $14(26)$ & $46(49)$ & $<0.01$ \\
\hline Current smoker $(n)$ & $7(13)$ & $14(15)$ & NS \\
\hline Prior smoker $(n)$ & $21(40)$ & $37(40)$ & NS \\
\hline Family history of coronary artery disease $(n)$ & $14(26)$ & $23(25)$ & NS \\
\hline \multicolumn{4}{|l|}{ LV function at rest } \\
\hline End-diastolic volume (mL) & $61.2 \pm 17.2$ & $63.6 \pm 17.3$ & NS \\
\hline End-systolic volume (mL) & $16.8 \pm 8.1$ & $19.8 \pm 9.2$ & NS \\
\hline Ejection fraction $(\%)$ & $74.1 \pm 8.8$ & $70.0 \pm 8.3$ & 0.01 \\
\hline Transient ischemic dilatation & $1.0 \pm 0.1$ & $1.0 \pm 0.1$ & NS \\
\hline \multicolumn{4}{|l|}{ LV phase analysis } \\
\hline Phase SD $\left(^{\circ}\right)$ & $10.2 \pm 4.0$ & $10.5 \pm 3.9$ & NS \\
\hline Histogram bandwidth $\left(^{\circ}\right)$ & $37.8 \pm 16.2$ & $40.5 \pm 17.4$ & NS \\
\hline PSS index & $5.6 \pm 5.1$ & $9.8 \pm 10.2$ & $<0.01$ \\
\hline SSS & $3.8 \pm 3.2$ & $7.0 \pm 4.8$ & $<0.01$ \\
\hline SRS & $1.7 \pm 2.0$ & $2.2 \pm 3.0$ & NS \\
\hline SDS & $2.1 \pm 2.9$ & $4.8 \pm 3.8$ & $<0.01$ \\
\hline
\end{tabular}

Data are expressed as mean \pm SD or as number, with percentage in parentheses.

NS $=$ not significant.

parameters, phase SD and histogram bandwidth, between the subjects with significant coronary artery stenosis and those without. Conversely, the PSS index was significantly higher in patients with coronary artery disease than in those without (Fig. 2). There were significant correlations between PSS index and phase SD and histogram bandwidth (Fig. 3). Representative phase analysis and 17-segment thickening curves are shown in Figure 4.
The optimal cutoff points for the phase SD, histogram bandwidth, PSS index, and SDS for the detection of coronary artery stenosis were determined using the ROC analysis. The optimal cutoff points were $10^{\circ}$ for phase SD, $37^{\circ}$ for histogram bandwidth, 6 for PSS index, 4 for SSS, 2 for SRS, and 3 for SDS. The areas under the ROC curves were $0.53,0.53,0.64,0.71,0.52$, and 0.74 , respectively. The PPV of a PSS index greater than 6 was $76 \%$ (95\% confidence interval, 68\%84\%). The sensitivity, specificity, PPVs, NPVs, and accuracy of the cutoff points of the 4 parameters are summarized in Table 2. The diagnostic value of the combination of the PSS index and SDS is shown in Figure 5.

Among the 9 parameters tested, the PSS index, SSS and SDS, but not phase SD or histogram bandwidth, and SRS were significantly associated with coronary artery stenosis on univariate analysis. The PSS index and SDS remained significant independent predictors for coronary artery disease on the multivariate analysis adjusted for sex and age (Table 3).

In addition, to detect multivessel disease, the area under the ROC curve was 


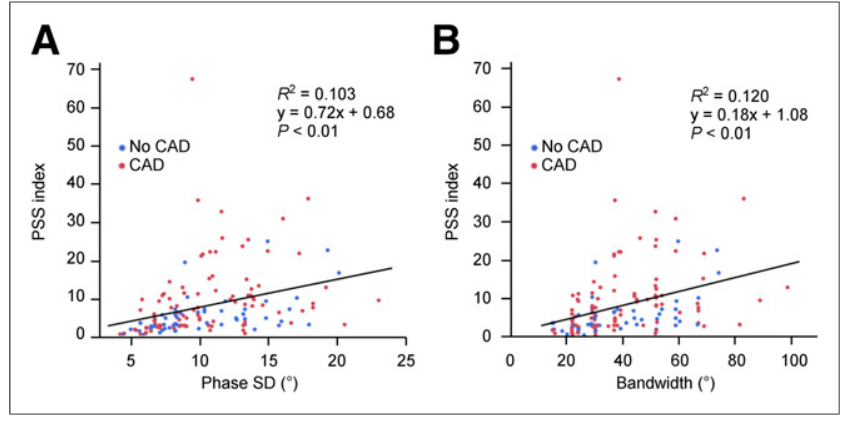

FIGURE 3. Comparisons of PSS index with phase SD and histogram bandwidth. $\mathrm{CAD}=$ coronary artery disease.

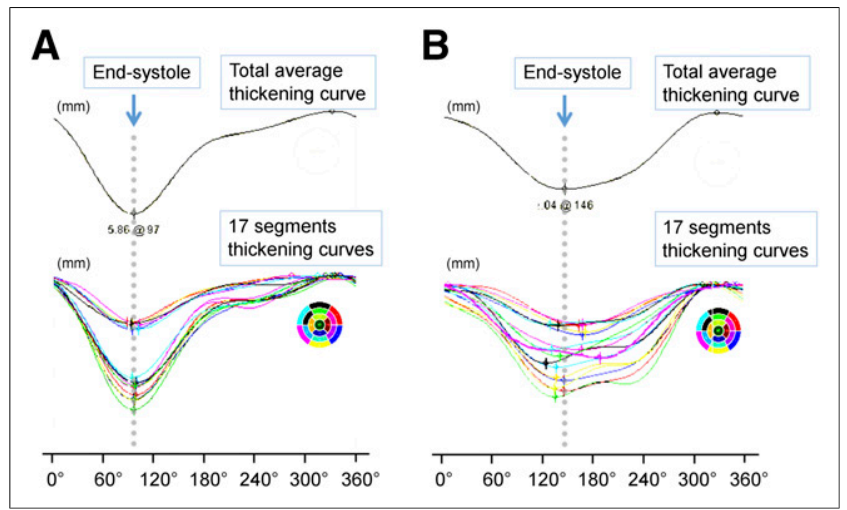

FIGURE 4. Representative cases of phase analysis and 17-segment thickening curves. Results from patient without coronary artery stenosis (A) and results from patient with significant coronary artery stenosis of right coronary artery and left circumflex artery (B). In A, phase $S D=10^{\circ}$, histogram bandwidth $=37^{\circ}$, and PSS index $=2.7$, and in B, phase $\mathrm{SD}=10^{\circ}$, histogram bandwidth $=39^{\circ}$, and PSS index $=29.6$.

higher for the PSS index than for transient ischemic dilatation (0.57 vs. 0.49). The diagnostic accuracy of a PSS index greater than 6 was as follows: sensitivity, 58\%; specificity, 58\%; PPV, $31 \%$; and NPV, $81 \%$.

\section{DISCUSSION}

The results of the current study demonstrated that the extent of the PSS index evaluated using segmental thickening curves extracted from QGS data in the resting condition was associated with the presence of coronary artery stenosis among the patients with suspected or confirmed coronary artery disease.
The QGS method is widely used to assess LV function $(1,4,5)$. Phase analysis of QGS images was developed to assess LV dyssynchrony (6-8). The phase SD and histogram bandwidth are associated with the prognosis of patients with LV dysfunction (7-13). In addition, several studies have also investigated the value of these parameters from alterations in the stress-rest image for the diagnosis of coronary artery disease (14-16). Al Jaroudi et al. reported that even the presence of a large reversible perfusion defect did not alter the indices of mechanical dyssynchrony (14). In contrast, Chen et al. reported a significant increase in postdipyridamole ${ }^{201}$ tallium stress-rest alternation in patients with ischemia, and using 99m Tc-sesamibi, Hida et al. also showed significant increases in postexercise stress LV dyssynchrony in patients with multivessel coronary artery disease $(13,16)$. The differences in the findings of these 3 studies may have originated from the timing of the acquired SPECT images (images are usually acquired 5-60 min after stress, not during stress), the different kinds of stress and radiotracers, and the dose of radiotracer. Furthermore, phase analysis may reflect many different factors throughout the cardiac cycle.

In the present study, the PSS index was derived using QGS from 17-segment thickening curves acquired during rest. This approach was selected because PSS is a sensitive and early sign of acute and chronic myocardial ischemia based on experimental and clinical data $(13,14,18-20)$. Asynergy of the left ventricle induced by stress generally improved soon. In contrast, PSS persisted longer than systolic disturbance, and the progress of PSS differed depending on the degree of stenosis $(20,25)$. In the present study, the patients with a higher PSS index were more likely to have coronary artery disease, and multivariate analysis showed that the PSS index was more strongly associated with coronary artery disease than the other dyssynchrony indices, phase SD and histogram bandwidth.

The mechanism underlying PSS has not been clearly addressed by previous studies. Patients with severe coronary artery disease may suffer ischemia even in daily life, and PSS persists after stress because of prolonged stunning or myocardial hibernation. In the present study, PSS assessed by ${ }^{99 m}$ Tc-tetrofosmin QGS demonstrated different dyssynchrony patterns between the patients with and without significant coronary stenosis, even at rest. In general, it is difficult to diagnose ischemia without any provocation. Dilsizian et al. reported persistent abnormalities of fatty acid metabolism $30 \mathrm{~h}$ after transient ischemia using ${ }^{123}$ I-BMIPP imaging (28). In addition, PSS and diastolic index acquired at rest using tissue Doppler imaging echocardiography have been shown to predict coronary artery disease with a sensitivity of $60 \%-83 \%$ and specificity 74\%-90\% (23). Here, we demonstrated that PSS evaluated using QGS could predict coronary artery disease. This method can

TABLE 2

Diagnostic Accuracy for Detecting Coronary Artery Disease

\begin{tabular}{lccccc}
\hline \multicolumn{1}{c}{ Predictor } & Area under curve & Sensitivity (\%) & Specificity (\%) & PPV (\%) & NPV (\%) \\
\hline Phase SD & 0.53 & 48 & 57 & 66 & 38 \\
\hline Histogram bandwidth & 0.53 & 51 & 59 & 68 & 40 \\
PSS index & 0.64 & 55 & 70 & 76 & 47 \\
SSS & 0.71 & 74 & 55 & 54 & 54 \\
SRS & 0.52 & 45 & 55 & 79 & 36 \\
SDS & 0.74 & 74 & 66 & 59 \\
\hline
\end{tabular}




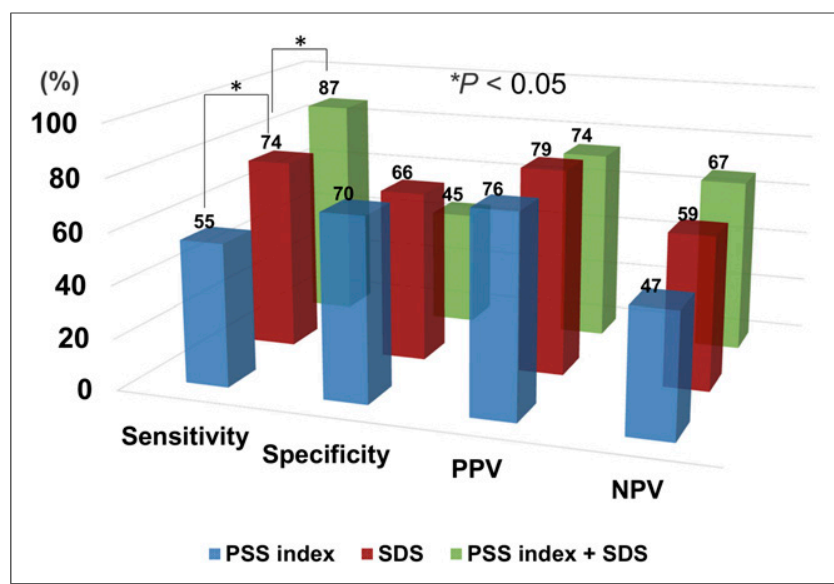

FIGURE 5. Diagnostic usefulness of PSS index, SDS, and combination of PSS index and SDS.

be performed in an automated manner during a relatively short period of time and with high reproducibility.

This study has some limitations. First, the study was conducted retrospectively at a single center with a small number of participants. Therefore, a larger cohort of prospectively selected patients would strengthen the findings. Second, we did not assess dyssynchrony variables using other modalities because all the patients had normal LV function without heart failure. Third, there was a technical limitation in that data were acquired at 16 frames per cardiac cycle and end-systole as average systole LV thickening, but not aortic valve closure, which may have decreased the PSS index. Acquisition at higher frames per cardiac cycle is theoretically better to detect PSS. Fourth, we did not investigate the relationship between the selected parameters and the degree of stenosis of the coronary artery. Fifth, PSS is not specific for ischemia because it can occur in hypertensive heart disease, hypertrophic cardiomyopathy, dilated cardiomyopathy, left bundle branch block, and normal hearts $(20,29,30)$. Sixth, this study excluded patients with prior myocardial infarction because the QGS method has limited ability to evaluate LV thickening in areas of low perfusion. Finally, the diagnostic criteria of coronary stenosis included stenosis of the coronary arteries based on angiographic findings, rather than physiologic changes, such as the functional flow reserve or coronary flow reserve.

\section{Potential Clinical Implications and Future Directions}

Today, QGS is the standard imaging technique used to assess LV function in clinical settings. Segmental thickening curves can be generated in a few extra minutes. The patients with higher PSS index were more likely to have coronary artery disease, and lower PSS index was associated with lower prevalence of multivessel disease. These results suggest that the patients suffer ischemia even at rest, and this contributes to prognosis. Further large-scale, longterm studies are required to better understand the diagnostic and prognostic value of PSS. The technique was semiautomated, nonuser-dependent, coupled with simultaneous myocardial perfusion assessment and 3-dimensional analyses from resting QGS scan.

\section{CONCLUSION}

The results of the present study indicate that PSS index at rest assessed using QGS might be a clinically useful indicator for detection of coronary artery disease in patients with diagnosed or suspected coronary artery disease but with no visual evidence of regional wall motion abnormality.

\section{DISCLOSURE}

The costs of publication of this article were defrayed in part by the payment of page charges. Therefore, and solely to indicate this fact, this article is hereby marked "advertisement" in accordance with 18 USC section 1734 . No potential conflict of interest relevant to this article was reported.

\section{ACKNOWLEDGMENTS}

We are thankful for the technical assistance of the staff (Akira Asazu and Hideyuki Sodeoka) in our nuclear medicine department; the secretarial assistance of Yuko Takenaka and Tomoko Takabayashi; and the technical advice of Yoshihiro Nishimura, Kenji Kuse, and Masahiro Kubota.

TABLE 3

Univariate and Multivariate Logistic Analysis for Detecting Coronary Artery Stenosis

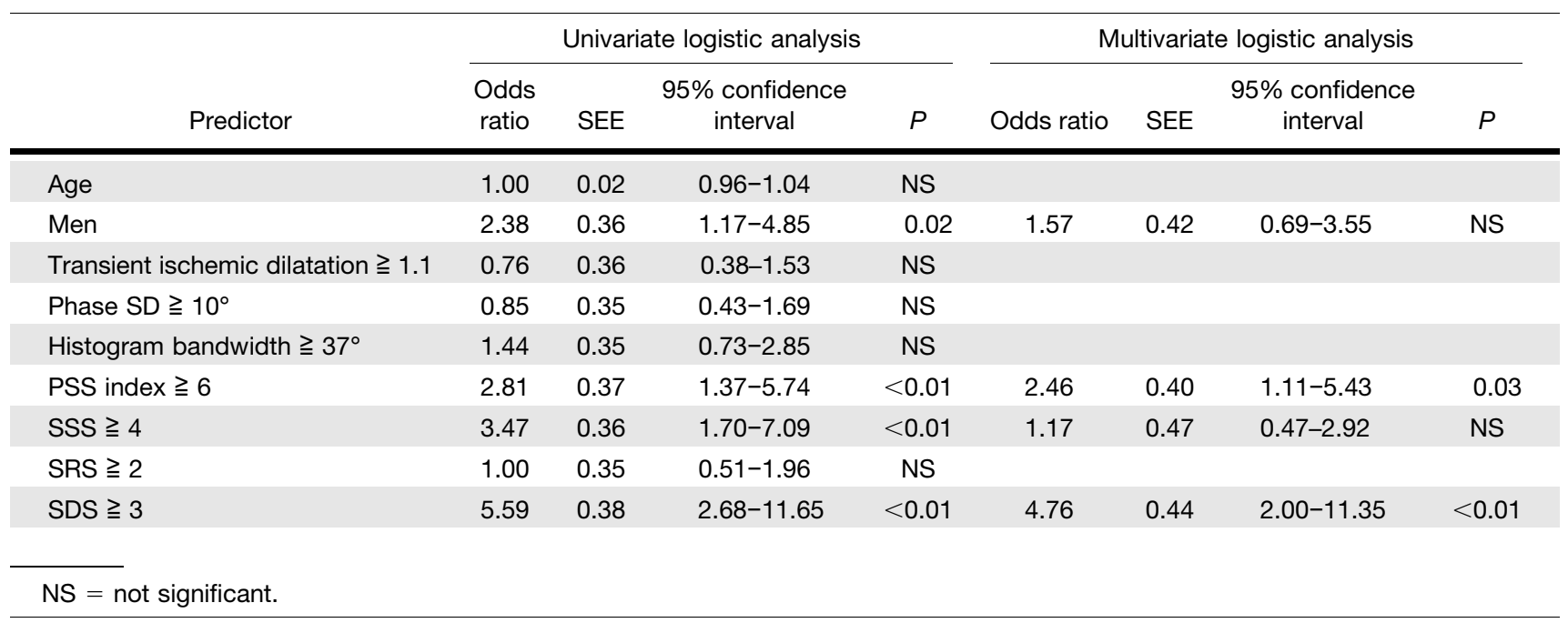




\section{REFERENCES}

1. Germano G, Kiat H, Kavanagh PB, et al. Automatic quantification of ejection fraction from gated myocardial perfusion SPECT. J Nucl Med. 1995;36:21382147.

2. Smanio PE, Watson DD, Segalla DL, Vinson EL, Smith WH, Beller GA. Value of gating of technetium-99m sestamibi single-photon emission computed tomographic imaging. J Am Coll Cardiol. 1997;30:1687-1692.

3. Sharir T, Germano G, Kavanagh PB, et al. Incremental prognostic value of poststress left ventricular ejection fraction and volume by gated myocardial perfusion single photon emission computed tomography. Circulation. 1999;100:1035-1042.

4. Akincioglu C, Berman DS, Nishina H, et al. Assessment of diastolic function using 16-frame ${ }^{99 \mathrm{~m}} \mathrm{Tc}$-sestamibi gated myocardial perfusion SPECT: normal values. J Nucl Med. 2005;46:1102-1108.

5. Bavelaar-Croon CD, Pauwels EK, van der Wall EE. Gated single-photon emission computed tomographic myocardial imaging: a new tool in clinical cardiology. Am Heart J. 2001;141:383-390.

6. Germano G, Kavanagh PB, Slomka PJ, Van Kriekinge SD, Pollard G, Berman DS. Quantitation in gated perfusion SPECT imaging: the Cedars-Sinai approach. J Nucl Cardiol. 2007;14:433-454.

7. Henneman MM, Chen J, Dibbets-Schneider P, et al. Can LV dyssynchrony as assessed with phase analysis on gated myocardial perfusion SPECT predict response to CRT? J Nucl Med. 2007;48:1104-1111.

8. AlJaroudi W, Jaber WA, Cerqueira MD. Effect of tracer dose on left ventricular mechanical dyssynchrony indices by phase analysis of gated single photon emission computed tomography myocardial perfusion imaging. J Nucl Cardiol. 2012; 19:63-72.

9. Van Kriekinge SD, Nishina H, Ohba M, Berman DS, Germano G. Automatic global and regional phase analysis from gated myocardial perfusion SPECT imaging: application to the characterization of ventricular contraction in patients with left bundle branch block. J Nucl Med. 2008;49:1790-1797.

10. Pazhenkottil AP, Buechel RR, Husmann L, et al. Long-term prognostic value of left ventricular dyssynchrony assessment by phase analysis from myocardial perfusion imaging. Heart. 2011;97:33-37.

11. Uebleis C, Hellweger S, Laubender RP, et al. Left ventricular dyssynchrony assessed by gated SPECT phase analysis is an independent predictor of death in patients with advanced coronary artery disease and reduced left ventricular function not undergoing cardiac resynchronization therapy. Eur J Nucl Med Mol Imaging. 2012;39:1561-1569.

12. Uebleis C, Hoyer X, Van Kriekinge SD, et al. Association between left ventricular mechanical dyssynchrony with myocardial perfusion and functional parameters in patients with left bundle branch block. J Nucl Cardiol. 2013;20:253-261.

13. Chen J, Garcia EV, Bax JJ, Iskandrian AE, Borges-Neto S, Soman P. SPECT myocardial perfusion imaging for the assessment of left ventricular mechanical dyssynchrony. J Nucl Cardiol. 2011;18:685-694.

14. Aljaroudi W, Koneru J, Heo J, Iskandrian AE. Impact of ischemia on left ventricular dyssynchrony by phase analysis of gated single photon emission computed tomography myocardial perfusion imaging. J Nucl Cardiol. 2011;18:36-42.

15. Chen CC, Shen TY, Chang MC, et al. Stress-induced myocardial ischemia is associated with early post-stress left ventricular mechanical dyssynchrony as assessed by phase analysis of ${ }^{201} \mathrm{Tl}$ gated SPECT myocardial perfusion imaging. Eur J Nucl Med Mol Imaging. 2012;39:1904-1909.

16. Hida S, Chikamori T, Tanaka H, et al. Diagnostic value of left ventricular dyssynchrony after exercise and at rest in the detection of multivessel coronary artery disease on single-photon emission computed tomography. Circ J. 2012;76: 1942-1952.

17. Huang WS, Huang CH, Lee CL, Chen CP, Hung GU, Chen J. Relation of early post-stress left ventricular dyssynchrony and the extent of angiographic coronary artery disease. J Nucl Cardiol. 2014;21:1048-1056.

18. Takayama M, Norris RM, Brown MA, Armiger LC, Rivers JT, White HD. Postsystolic shortening of acutely ischemic canine myocardium predicts early and late recovery of function after coronary artery reperfusion. Circulation. 1988;78:9941007.

19. Wang J, Abraham TP, Korinek J, Urheim S, McMahon EM, Belohlavek M. Delayed onset of subendocardial diastolic thinning at rest identifies hypoperfused myocardium. Circulation. 2005;111:2943-2950.

20. Voigt JU, Exner B, Schmiedehausen K, et al. Strain-rate imaging during dobutamine stress echocardiography provides objective evidence of inducible ischemia. Circulation. 2003;107:2120-2126.

21. Kukulski T, Jamal F, Herbots L, et al. Identification of acutely ischemic myocardium using ultrasonic strain measurements: a clinical study in patients undergoing coronary angioplasty. J Am Coll Cardiol. 2003;41:810-819.

22. Bijnens B, Claus P, Weidemann F, Strotmann J, Sutherland GR. Investigating cardiac function using motion and deformation analysis in the setting of coronary artery disease. Circulation. 2007;116:2453-2464.

23. Onishi T, Uematsu M, Nanto S, et al. Detection of diastolic abnormality by dyssynchrony imaging: correlation with coronary artery disease in patients presenting with visibly normal wall motion. Circ J. 2009;73:125-131.

24. Ryf S, Rutz AK, Boesiger P, Schwitter J. Is post-systolic shortening a reliable indicator of myocardial viability? An MR tagging and late-enhancement study. $J$ Cardiovasc Magn Reson. 2006;8:445-451.

25. Asanuma T, Uranishi A, Masuda K, Ishikura F, Beppu S, Nakatani S. Assessment of myocardial ischemic memory using persistence of post-systolic thickening after recovery from ischemia. JACC Cardiovasc Imaging. 2009;2:1253-1261.

26. Asanuma T, Nakatani S. Myocardial ischaemia and post-systolic shortening. Heart. 2015;101:509-516.

27. Austen WG, Edwards JE, Frye RL, et al. A reporting system on patients evaluated for coronary artery disease: report of the Ad Hoc Committee for Grading of Coronary Artery Disease, Council on Cardiovascular Surgery, American Heart Association. Circulation. 1975;51:5-40.

28. Dilsizian V, Bateman TM, Bergmann SR, et al. Metabolic imaging with betamethyl-p-[ $\left.{ }^{123} \mathrm{I}\right]$-iodophenyl-pentadecanoic acid identifies ischemic memory after demand ischemia. Circulation. 2005;112:2169-2174.

29. Weidemann F, Broscheit JA, Bijnens B, et al. How to distinguish between ischemic and nonischemic postsystolic thickening: a strain rate imaging study. Ultrasound Med Biol. 2006;32:53-59.

30. Pislaru C, Anagnostopoulos PC, Seward JB, Greenleaf JF, Belohlavek M. Higher myocardial strain rates during isovolumic relaxation phase than during ejection characterize acutely ischemic myocardium. J Am Coll Cardiol. 2002;40:14871494. 